This item was submitted to Loughborough's Research Repository by the author.

Items in Figshare are protected by copyright, with all rights reserved, unless otherwise indicated.

\title{
On the actuator dynamics of dynamic control allocation for a small fixed-wing UAV with direct lift control
}

\author{
PLEASE CITE THE PUBLISHED VERSION
}

https://doi.org/10.1109/TCST.2019.2945909

\section{PUBLISHER}

Institute of Electrical and Electronics Engineers

\section{VERSION}

AM (Accepted Manuscript)

\section{PUBLISHER STATEMENT}

(C) 2019 IEEE. Personal use of this material is permitted. Permission from IEEE must be obtained for all other uses, in any current or future media, including reprinting/republishing this material for advertising or promotional purposes, creating new collective works, for resale or redistribution to servers or lists, or reuse of any copyrighted component of this work in other works.

\section{LICENCE}

\section{All Rights Reserved}

\section{REPOSITORY RECORD}

Yan, Yunda, Jun Yang, Cunjia Liu, Matthew Coombes, Shihua Li, and Wen-Hua Chen. 2020. "On the Actuator Dynamics of Dynamic Control Allocation for a Small Fixed-wing UAV with Direct Lift Control". Loughborough University. https://hdl.handle.net/2134/9926891.v1. 


\title{
On the Actuator Dynamics of Dynamic Control Allocation for a Small Fixed-wing UAV with Direct Lift Control
}

\author{
Yunda Yan, Member, IEEE, Jun Yang, Senior Member, IEEE, Cunjia Liu, Member, IEEE, \\ Matthew Coombes, Shihua Li, Senior Member, IEEE, Wen-Hua Chen, Fellow, IEEE
}

\begin{abstract}
A novel dynamic control allocation method is proposed for a small fixed-wing unmanned aerial vehicle (UAV), whose flaps can be actuated as fast as other control surfaces, offering an extra way of changing the lift directly. The actuator dynamics of this kind of UAVs, which may be sluggish comparing to the UAV dynamics, should also be considered in the control design. To this end, a hierarchical control allocation architecture is developed. A disturbance observer based high-level tracking controller is first designed to accommodate the lagging effect of the actuators and to compensate the adverse effect of external disturbances. Then, a dynamic control allocator based on a receding-horizon performance index is developed, which forces the actuator state in the low-level to follow the optimised reference. Compared to the conventional control allocation method that assumes ideal actuators with infinite bandwidths, higher tracking accuracy of the UAV and better energy efficiency can be achieved by the proposed method. Stability analysis and high fidelity simulations both demonstrate the effectiveness of the proposed method, which can be deployed on different fixed-wing UAVs with flaps to achieve better performance.
\end{abstract}

Index Terms-Control allocation, actuator dynamics, disturbance observer, non-minimum phase, flight control.

\section{INTRODUCTION}

$\mathbf{R}$ ECENT decades have witnessed the rapid growth on both the development and application of small unmanned aerial vehicles (UAVs) in different domains, ranging from surveillance, payload delivery to environmental monitoring and agriculture mapping. Among different UAV configurations, such as helicopters and multicopters, conventional fixedwing UAVs still manage to position themselves in many critical applications, because their simpler structure and better aerodynamic efficiency can offer longer endurance and higher airspeed. For many small fixed-wing UAVs, they are normally featured with low-cost and light-weight designs to facilitate the rapid deployment to various applications. However, these attributes also mean that their limited power and low inertia

This work was supported in part by the UK EPSRC Grant EP/P012868/1, in part by the National Natural Science Foundation of China under Grant Nos. 61973080, 61973081 and 61750110525, in part by the State Scholarship Fund under Grant No. 201706090111. (Corresponding authors: Cunjia Liu and Shihua Li.)

Y. Yan, J. Yang, and S. Li are with the Key Laboratory of Measurement and Control of CSE, Ministry of Education, School of Automation, Southeast University, Nanjing 210096, China, E-mail: (yd.yan@ieee.org, yyd@seu.edu.cn; j.yang84@seu.edu.cn; lsh@seu.edu.cn).

C. Liu, M. Coombes, and W.-H. Chen are with the Department of Aeronautical and Automotive Engineering, Loughborough University, Loughborough LE11 3TU, U.K., E-mail: (c.liu5@lboro.ac.uk; m.j.coombes@lboro.ac.uk; w.chen@lboro.ac.uk). may make them susceptible to external wind gusts, leading to degraded flight performance. To alleviate these phenomena, some control oriented techniques [1]-[4] have been investigated to compensate the wind disturbances.

This paper approaches this problem from a different perspective, specifically by exploiting the flight mechanism of conventional fixed-wing UAVs equipped with dedicated flaps. Flaps can be used to change the lift and drag, which is often referred to as direct lift control (DLC) in aviation literature [5]. Unlike their counterparts on full-size aircraft, the flaps on small UAVs are usually actuated by the same electric servos used for other control surfaces. This means that the flaps can be used as ordinary control surfaces in the same way as elevators, which in turn, provides the control redundancy and extra control authority to deal with external disturbances. Therefore, to fully explore the potential of DLC on small fixed-wing UAVs, a solution to control allocation is required to distribute the desired total control effort among a redundant set of actuators, i.e. the elevator, motor thrust, and flap.

Various control allocation methods, e.g., pseudo-inverse, daisy chaining and direct allocation, have been developed and extensively investigated in literature [6]-[9], some of which have a particular focus on reconfigurable flight control design [10]-[12]. Apart from the basic control allocation functions, many advanced algorithms have considered additional factors, e.g., actuator energy saving and actuator safety, using techniques such as linear/nonlinear constrained quadratic programming [13], [14], additional dynamic augmentation [15] and input matrix factorization [16]. However, those control allocation methods may not be readily applicable to the problem investigated in this work due to the presence of slow actuator dynamics. It is very common in control allocation to assume ideal actuators with infinite bandwidths, ignoring their dynamics. This assumption is justifiable since the dynamics of many over-actuated systems, e.g., cargo ship, full-size aircraft and submarine, are indeed much slower than those of their actuators. On the contrary, the actuator dynamics of small fixed-wing UAVs cannot be ignored in high-performance flight control due to the agile dynamics of a small UAV.

Several pioneering works on control allocation with the consideration of actuator dynamics can be found in literature. In [9, Ch. 7], the existence of actuator dynamics is regarded as one of the most significant obstacles in control allocation, because the input matrix of the cascaded system can not be factorized into two matrices with lower dimensions, suggesting 
that the conventional control allocation methods cannot be applied directly. To the best of the authors' knowledge, the first viable solution was proposed in [17] by introducing a punishment on the allocation rate. This approach is also referred to as dynamic control allocation, which indicates that an additional transient process is introduced for control allocation. Hence, it is easier for actuators to catch up with the time-varying control signals. Following this dynamic approach, some important progress has been made in both theory [18] and applications [19]. However, it is worth noting that this kind of dynamic allocation methods only relieves the adverse effect of actuator dynamics, because the perfect allocation is only available for constant control signals (see [17, Thm. 3] for details). On the other hand, a practical approach to compensate the actuator dynamics via the measurement of actuator states is proposed in [20]. However, since it is an open loop compensation, only very fast sampling rate can guarantee the effectiveness and the stability of the closed-loop system, setting high requirements on the sensors of actuators. In [21], model predictive control (MPC) method is used for control allocation, which considers a second order actuator model with constraints on position and velocity. In [22], an adaptive control allocation method is proposed for stabilization of a much more general case where the actuator model is not only nonlinear but also with parametric uncertainties, which increases the complexity for practical tracking applications. Meanwhile, along the line of linear full-information output regulation for over-actuated systems, the rigorous theoretical framework has been build up in [23]-[27]. Specifically, on the basis of [24], over-actuated plants with parametric uncertainties are considered in [25] whilst in [26], [27], MPC methods are involved to specify desired steady states with constraints. These methods have the potential to deal with the problem considered in this paper by several extensions and embedding the actuator dynamics into the considered system.

To tackle the challenges on DLC based control for small fixed-wing UAVs, namely, disturbance rejection, actuator dynamics and control allocation, a novel integrated control design with dynamic control allocation is proposed in this work. The considered actuators are of linear dynamics without constraints, but their dynamic states are unmeasurable. Following the structure of conventional control allocation [13], a hierarchical framework is adopted to pursue the superiorities of modular design. The structure of the proposed framework consists of two parts. In the high level, a linear control design with a compensator is adopted for precise output tracking. Due to the insufficient sensors for actuators and external disturbances, a disturbance observer [28] is combined with the tracking controller to achieve the objectives of output tracking and disturbance rejection. However, embedding the actuator dynamics into the high-level tracking design will introduce additional zero dynamics into the system. Therefore, the corresponding low-level allocator needs to be designed not only to reduce the extra energy consumption caused by control inputs, but also to stabilize the internal dynamics [29], [30]. In the low level, inspired by the generalized predictive control method [31], a receding-horizon performance index is adopted to represent the total cost. Unlike the conventional performance indexes in control allocation (e.g., $J(t)=\|W u(t)\|_{1,2, \infty}$ ), which is only related with the current control input, the receding-horizon one contains the future information, and hence, is dynamic. By using the Taylor expansion for prediction, the optimal desired states of actuators are explicitly obtained, which are directly dependent on the disturbance estimates in the high level and the reference commands of the UAV. Subsequently, a virtual allocator is designed to force the actuator states to the optimal ones.

Notation: For any smooth enough function $f(t)$, symbol $f^{(i)}(t)$ denotes the $i$-th order derivative of $f(t)$ with respect to variable $t$. For any state $x$, symbols $\hat{x}$ and $x_{r}$ denote its estimate and reference, respectively. For any matrix $\boldsymbol{A} \in \mathbb{R}^{m \times n}$, $\boldsymbol{A}_{(i, j)} \in \mathbb{R}$ denotes the element in $i$-th row and $j$-th column of $\boldsymbol{A}, \boldsymbol{A}_{(i,:)} \in \mathbb{R}^{1 \times n}$ denotes the vector row $i$ of $\boldsymbol{A}$, and

$$
\begin{aligned}
\boldsymbol{A}_{(i: j,:)} & \triangleq\left[\begin{array}{llll}
\boldsymbol{A}_{(i,:)}^{T} & \boldsymbol{A}_{(i+\mathbf{1},:)}^{T} & \cdots & \boldsymbol{A}_{(j,:)}^{T}
\end{array}\right]^{T} \in \mathbb{R}^{(j-i+1) \times n} \\
\boldsymbol{A}_{(i: j, p)} & \triangleq\left[\begin{array}{llll}
\boldsymbol{A}_{(i, p)} & \boldsymbol{A}_{(i+1, p)} & \cdots & \boldsymbol{A}_{(j, \boldsymbol{p})}
\end{array}\right]^{T} \in \mathbb{R}^{(j-i+1) \times 1} \\
\boldsymbol{A}_{(i, p: q)} & \triangleq\left[\begin{array}{llll}
\boldsymbol{A}_{(i, p)} & \boldsymbol{A}_{(i, p+1)} & \cdots & \boldsymbol{A}_{(i, q)}
\end{array}\right] \in \mathbb{R}^{1 \times(q-p+1)} \\
\boldsymbol{A}_{(i: j, p: q)} & \triangleq\left[\begin{array}{llll}
\boldsymbol{A}_{(i: j, p)} & \boldsymbol{A}_{(i: j, p+1)} & \cdots & \boldsymbol{A}_{(i: j, q)}
\end{array}\right] \in \mathbb{R}^{(j-i+1) \times(q-p+1)}
\end{aligned}
$$

where $n, m, i, j, p, q \in \mathbb{N}_{+}, 1 \leq i \leq j \leq m$ and $1 \leq p \leq q \leq$ $n$. Matrix $\mathbf{0}_{i \times j}$ denotes an $i \times j$ zero matrix and matrix $\mathbf{1}_{k \times k}$ denotes a $k \times k$ identity matrix.

\section{Problem Formulation}

In this section, the linearized longitudinal model of a small fixed-wing UAV with the assistance of DLC, together with models of actuators, i.e., the elevator, motor throttle and flap, are briefly introduced. Readers can refer to [2], [32] for the illustration of the longitudinal dynamics of UAV.

\section{A. UAV Dynamics}

The linearized longitudinal model for the considered small fixed-wing UAV is given as follows [33, Ch. 5.5.3]:

$$
\dot{x}_{p}=A_{p} x_{p}+B_{p u} u_{p}+B_{p d} d, y_{p}=C_{p} x_{p}
$$

where $\boldsymbol{x}_{\boldsymbol{p}} \triangleq\left[\begin{array}{lllll}u & w & q & \theta & h\end{array}\right]^{T}, \boldsymbol{y}_{\boldsymbol{p}} \triangleq\left[\begin{array}{ll}u & h\end{array}\right]^{T}, \boldsymbol{u}_{\boldsymbol{p}} \triangleq\left[\begin{array}{lll}\delta_{e} & \delta_{t} & \delta_{f}\end{array}\right]^{T}$,

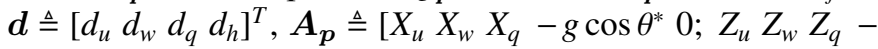

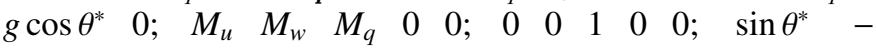
$\left.\cos \theta^{*} \quad 0 \quad u^{*} \cos \theta^{*}+w^{*} \sin \theta^{*} \quad 0\right], \quad B_{p u} \triangleq$ $\left[\begin{array}{lllllllllllllll}X_{\delta_{e}} & X_{\delta_{t}} & X_{\delta_{f}} ; & Z_{\delta_{e}} & 0 & Z_{\delta_{f}} ; & M_{\delta_{e}} & 0 & M_{\delta_{f}} ; & 0 & 0 & 0 ; & 0 & 0 & 0\end{array}\right]$, $\boldsymbol{B}_{\boldsymbol{p d}} \triangleq\left[\begin{array}{llllllllllllllllllll}1 & 0 & 0 & 0 ; & 0 & 1 & 0 & 0 ; & 0 & 0 & 1 & 0 ; & 0 & 0 & 0 & 0 ; & 0 & 0 & 0 & 1\end{array}\right]$,

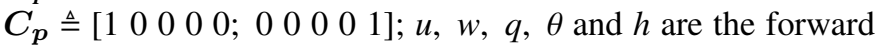
body velocity, vertical body velocity, pitch rate, pitch angle and height, respectively; $\delta_{e}, \delta_{t}$ and $\delta_{f}$ are the control inputs generated by the elevator, motor throttle and flap, respectively; $X, Z$ and $M$ are the force and moment coefficients given their associated subscripts; $g$ is the acceleration of gravity; any state denoted further with $*$ represents the state at the linearization point of the model; $d_{u}, d_{w}, d_{q}$ and $d_{h}$ are the lumped effects of external disturbances and directly affect the system states $u, w, q$ and $h$, respectively. Since most external disturbances in flight control systems, e.g., wind gusts, normally present much slower dynamics than UAV itself [2], $\boldsymbol{d}$ is therefore assumed to be unknown constant. 


\section{B. Actuator Dynamics}

The elevator and flap are directly driven by electric servos and are much faster than the motor thrust dynamics. Therefore, first-order systems are used to represent the actuator dynamics of the elevator and flap, whilst a second-order one is used for the motor thrust. The dynamics of the three actuators are written compactly as follows:

$$
\dot{x}_{a}=A_{a} x_{a}+B_{a} u_{a}, y_{a}=C_{a} x_{a}
$$

where $\boldsymbol{x}_{\boldsymbol{a}} \triangleq\left[\begin{array}{llll}\delta_{e} & \delta_{t} & \dot{\delta}_{t} & \delta_{f}\end{array}\right]^{T}, \boldsymbol{y}_{\boldsymbol{a}} \triangleq \boldsymbol{u}_{\boldsymbol{p}}, \boldsymbol{u}_{\boldsymbol{a}} \triangleq\left[\begin{array}{lll}u_{e} & u_{t} & u_{f}\end{array}\right]^{T}$,

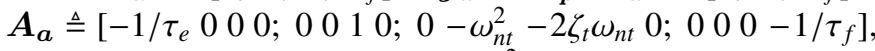
$\boldsymbol{B}_{\boldsymbol{a}} \triangleq\left[\begin{array}{lllllllllllll}1 / \tau_{e} & 0 & 0 ; & 0 & 0 & 0 ; & 0 & \omega_{n t}^{2} & 0 ; & 0 & 0 & 1 / \tau_{f}\end{array}\right], \boldsymbol{C}_{\boldsymbol{a}} \triangleq$

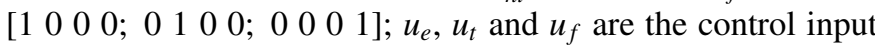
signals to the elevator, motor throttle and flap, respectively; $\tau_{e}$ and $\tau_{f}$ are the time constants of the elevator and flap, respectively; $\omega_{n t}$ and $\zeta_{t}$ are the oscillation frequency and damping ratio of the thrust dynamics, respectively. Combining the UAV dynamics, the following assumption is adopted, which can be easily verified for different UAV systems.

Assumption 1: $\left(A_{a}, B_{p u} C_{a}\right)$ is observable.

\section{Control Objective}

Let $\boldsymbol{x} \triangleq\left[\boldsymbol{x}_{p}{ }^{T} \boldsymbol{x}_{\boldsymbol{a}}{ }^{T}\right]^{T}$ and substitute (2) into (1) to obtain the following actuator-dynamics-based UAV system:

$$
\dot{x}=A x+B_{u} u_{a}+B_{d} d, y_{p}=C x
$$

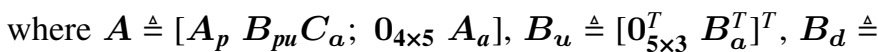
$\left[\begin{array}{ll}\boldsymbol{B}_{p \boldsymbol{d}}^{T} & \mathbf{0}_{\mathbf{4 \times 4}}^{T}\end{array}\right]^{T}$ and $\boldsymbol{C} \triangleq\left[\begin{array}{ll}\boldsymbol{C}_{\boldsymbol{p}} & \mathbf{0}_{\mathbf{2 \times 4}}\end{array}\right]$. The objective of this work is to design a control input $\boldsymbol{u}_{\boldsymbol{a}}$ such that the output of the UAV system $\boldsymbol{y}_{\boldsymbol{p}}$ asymptotically tracks any given sufficiently smooth and bounded reference $\boldsymbol{y}_{\boldsymbol{p r}} \triangleq\left[u_{r} h_{r}\right]^{T}$ under unknown external disturbances, and meanwhile, to save the total cost caused by the actuators. Before detailed design, the overall control scheme is presented in Fig. 1. Notably, the designed control input $\boldsymbol{u}_{\boldsymbol{a}}$ is only the input command to actuator, which is a virtual signal without much physical significance. Hence, the output of actuator $\boldsymbol{y}_{\boldsymbol{a}}$ (or $\boldsymbol{u}_{\boldsymbol{p}}$ ), rather than its input $\boldsymbol{u}_{\boldsymbol{a}}$, will be optimized in the subsequent allocation design.

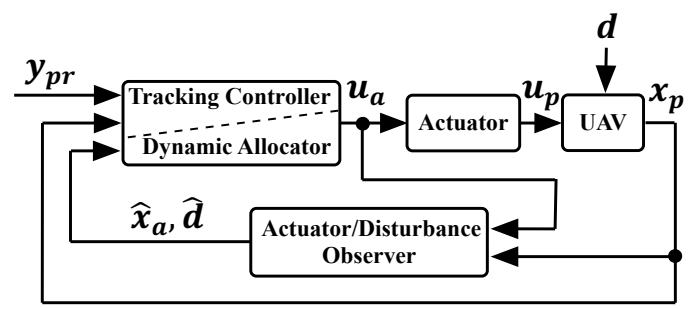

Fig. 1. Schematic diagram of the proposed method.

\section{Actuator/Disturbance-Observer-Based Tracking}

In this section, as shown in Fig. 2, the tracking controller in high level with a compensator for the lag effect of the internal actuators and the adverse effect of the external disturbances is designed for the augmented plant (3).

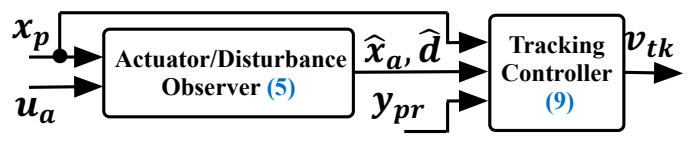

Fig. 2. Schematic diagram of the tracking controller in high level.

\section{A. Actuator-Dynamics-Based Disturbance Observer Design}

The designed observer should be able to estimate the actuator state $\boldsymbol{x}_{\boldsymbol{a}}$ as well as the external disturbance $\boldsymbol{d}$. With actuator dynamics (2), one arrives at

$$
\dot{x}_{a d}=A_{a d} x_{a d}+B_{a d} u_{a}, y_{a d}=C_{a d} x_{a d}
$$

where $x_{a d} \triangleq\left[\begin{array}{ll}x_{a}^{T} & d^{T}\end{array}\right]^{T}, y_{a d} \triangleq \dot{x}_{p}-A_{p} x_{p}, A_{a d} \triangleq$ $\left[\begin{array}{llll}A_{a} & \mathbf{0}_{4 \times 4} ; & \mathbf{0}_{4 \times 4} & \mathbf{0}_{4 \times 4}\end{array}\right], \boldsymbol{B}_{a d} \triangleq\left[\begin{array}{cc}B_{a}^{T} & \mathbf{0}_{4 \times 3}^{T}\end{array}\right]^{T}$ and $\boldsymbol{C}_{\boldsymbol{a d}} \triangleq$ $\left[\begin{array}{lll}\boldsymbol{B}_{p u} & \boldsymbol{C}_{\boldsymbol{a}} & \boldsymbol{B}_{p d}\end{array}\right]$. Note that $\left(\mathbf{0}_{\mathbf{4} \times \mathbf{4}}, \boldsymbol{B}_{\boldsymbol{p d}}\right)$ is observable. By Assumption 1, with Popov-Belevitch-Hautus observability criterion [34], one gets that $\left(\boldsymbol{A}_{\boldsymbol{a d}}, \boldsymbol{C}_{\boldsymbol{a d}}\right)$ is also observable. Following the approach in [35], by defining an internal state $\boldsymbol{z} \triangleq \hat{\boldsymbol{x}}_{\boldsymbol{a d}}-\boldsymbol{L} \boldsymbol{x}_{\boldsymbol{p}}$ with $\hat{\boldsymbol{x}}_{\boldsymbol{a d}} \triangleq\left[\begin{array}{ll}\hat{\boldsymbol{x}}_{\boldsymbol{a}}^{T} & \hat{\boldsymbol{d}}^{T}\end{array}\right]^{T}$, one gets the following actuator/disturbance observer:

$$
\begin{aligned}
\dot{z}= & \left(A_{a d}-L C_{a d}\right) z \\
& +\left(A_{a d} L-L A_{p}-L C_{a d} L\right) x_{p}+B_{a d} u_{a} \\
\hat{x}_{a d}= & z+L x_{p}
\end{aligned}
$$

where $\boldsymbol{L}$ is the observer gain.

\section{B. Tracking Controller Design Via Dynamic Inversion}

Based upon the parameters in Appendix A, the relative degree of each output is available as $C_{1} B_{u}=0, C_{1} A B_{u} \neq$ $0 ; C_{2} B_{u}=0, C_{2} A B_{u}=0, C_{2} A^{2} B_{u} \neq 0$, where $C_{1} \triangleq C_{(1, ;)}$ and $C_{2} \triangleq C_{(2, ;)}$. Since the sum of relative degrees is strictly less than the system dimension, there exists zero dynamics in (3). Following the approach in [36, Chap. 5.1], define a new state as

$$
\boldsymbol{D}_{\boldsymbol{X}} \triangleq\left[\begin{array}{ll}
\boldsymbol{D}_{\boldsymbol{Y}}^{T} & \boldsymbol{D}_{Z}^{T}
\end{array}\right]^{T}=\boldsymbol{T}_{\boldsymbol{M}} \boldsymbol{x}+\boldsymbol{T}_{\boldsymbol{N}} \boldsymbol{d}
$$

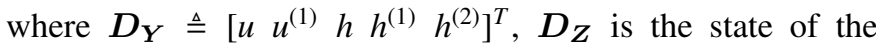
zero dynamics, and $T_{M}$ and $T_{N}$ are transformation matrices. To endow this coordinate transformation with more physical significance, let $D_{Z} \triangleq x_{a}$. The transformation matrices can then be fixed as follows:

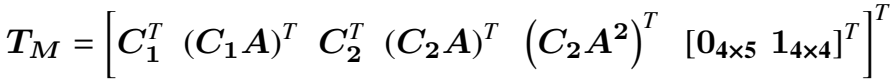

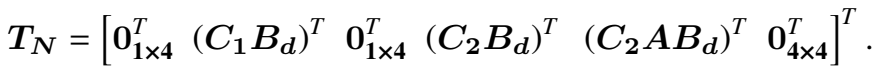

It is worth noting that $\operatorname{rank}\left(\boldsymbol{T}_{M}\right)=9$, which implies that mapping (6) is inverible between $\boldsymbol{D}_{\boldsymbol{X}}$ and $\boldsymbol{x}$. Taking derivatives of $\boldsymbol{D}_{\boldsymbol{X}}$ in (6) along (3) gives

$$
\dot{D}_{X}=A_{x} D_{X}+B_{x u} u_{a}+B_{x d} d
$$

where $A_{x} \triangleq T_{M} A T_{M}^{-1}, B_{x u} \triangleq T_{M} B_{u}$ and $B_{x d} \triangleq$ $-T_{M} A T_{M}^{-1} T_{N}+T_{M} B_{d}$. For tracking control design, extracting the second and the fifth lines from system (7) yields

$$
\left[\begin{array}{l}
u^{(2)} \\
h^{(3)}
\end{array}\right]=\left[\begin{array}{l}
\boldsymbol{A}_{\boldsymbol{x}(2,:)} \\
\boldsymbol{A}_{\boldsymbol{x}(5,:)}
\end{array}\right] \boldsymbol{D}_{\boldsymbol{X}}+\left[\begin{array}{c}
\boldsymbol{B}_{x d(2,:)} \\
\boldsymbol{B}_{x d(5,:)}
\end{array}\right] \boldsymbol{d}+\boldsymbol{v}_{\boldsymbol{t} k}
$$


where $\boldsymbol{v}_{\boldsymbol{t} \boldsymbol{k}} \triangleq\left[\begin{array}{ll}v_{u} & v_{h}\end{array}\right]^{T}=\boldsymbol{B}_{\boldsymbol{t} \boldsymbol{k}} \boldsymbol{u}_{a}$ and $\boldsymbol{B}_{\boldsymbol{t} \boldsymbol{}} \triangleq$ $\left[\left(\boldsymbol{C}_{\mathbf{1}} \boldsymbol{A} \boldsymbol{B}_{\boldsymbol{u}}\right)^{T}\left(\boldsymbol{C}_{\mathbf{2}} \boldsymbol{A}^{\mathbf{2}} \boldsymbol{B}_{\boldsymbol{u}}\right)^{T}\right]^{T}$. Using the dynamic inversion approach [37] gives the following tracking controller:

$$
\begin{aligned}
& v_{u}=\boldsymbol{K}_{\boldsymbol{u}}\left(\boldsymbol{D}_{\boldsymbol{Y r}(\mathbf{1 : 2 , 1 )}}-\hat{\boldsymbol{D}}_{\boldsymbol{Y}(\mathbf{1 : 2 , 1})}\right)+v_{u r} \\
& v_{h}=\boldsymbol{K}_{\boldsymbol{h}}\left(\boldsymbol{D}_{\boldsymbol{Y r}(\mathbf{3 : 5 , 1})}-\hat{\boldsymbol{D}}_{\boldsymbol{Y}(\mathbf{3 : 5 , 1})}\right)+v_{h r}
\end{aligned}
$$

where $\boldsymbol{D}_{\boldsymbol{Y} \boldsymbol{r}} \triangleq\left[\begin{array}{lllll}u_{r} & u_{r}^{(1)} & h_{r} & h_{r}^{(1)} & h_{r}^{(2)}\end{array}\right]^{T}, \hat{\boldsymbol{D}}_{\boldsymbol{X}} \triangleq \boldsymbol{T}_{\boldsymbol{M}}\left[\begin{array}{ll}\boldsymbol{x}_{\boldsymbol{p}}{ }^{T} & \hat{\boldsymbol{x}}_{\boldsymbol{a}}^{T}\end{array}\right]^{T}+$ $\boldsymbol{T}_{\boldsymbol{N}} \hat{d}=\left[\begin{array}{ll}\hat{D}_{\boldsymbol{Y}}^{T} & \hat{D}_{Z}^{T}\end{array}\right]^{T}, v_{u r} \triangleq u_{r}^{(2)}-\boldsymbol{A}_{\boldsymbol{x}(\mathbf{2}, \mathbf{)})} \hat{\boldsymbol{D}}_{\boldsymbol{X}}-\boldsymbol{B}_{\boldsymbol{x} d(\mathbf{2}, \mathbf{)})} \hat{d}, v_{h r} \triangleq$ $h_{r}^{(3)}-\boldsymbol{A}_{\boldsymbol{x}(\mathbf{5},:)} \hat{D}_{\boldsymbol{X}}-\boldsymbol{B}_{\boldsymbol{x}(\mathbf{5},:)} \hat{\boldsymbol{d}} ; \boldsymbol{K}_{\boldsymbol{u}}$ and $\boldsymbol{K}_{\boldsymbol{h}}$ are the controller gains.

Remark 1: There are two deficiencies of using the conventional control allocation method for allocation of the designed controller (9). On one hand, the targeted control input $\boldsymbol{u}_{\boldsymbol{a}}$ is a virtual signal without much physical significance. One the other hand, based upon the system parameters in Appendix

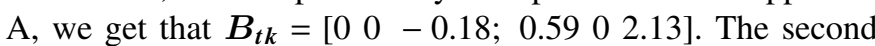
column of the pseudo-inverse of $\boldsymbol{B}_{\boldsymbol{t} \boldsymbol{k}}$ is $\mathbf{0}_{\mathbf{2} \times \mathbf{1}}$, which implies that $u_{t}^{*}=\boldsymbol{u}_{\boldsymbol{a}(\mathbf{2 , 1})}^{*}=0$ holds for any control signal $\boldsymbol{v}_{\boldsymbol{t} \boldsymbol{k}}$ generated from high level. Therefore, it will lose the freedom to stabilize the zero dynamics.

\section{Actuator-Dynamics-Based Dynamic Control Allocation}

In this section, the dynamic allocator in the low level considering the actuator dynamics is designed as shown in Fig. 3, following the stability analysis of the closed-loop system.

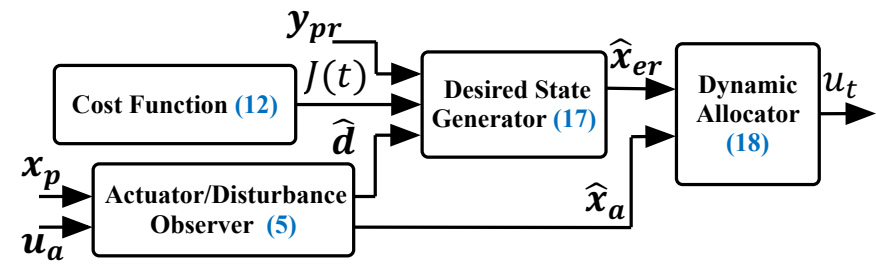

Fig. 3. Schematic diagram of the dynamic allocator in low level.

\section{A. Preliminaries}

Since that $\boldsymbol{B}_{\boldsymbol{t} \boldsymbol{k}} \in \mathbb{R}^{2 \times 3}$ with $\operatorname{rank}\left(\boldsymbol{B}_{\boldsymbol{t} \boldsymbol{k}}\right)=2$, it has a nullspace of dimension $1=3-2$, in which the control input $\boldsymbol{u}_{a}$ can be perturbed without affecting $\dot{\boldsymbol{D}}_{\boldsymbol{Y}}$. For convenience, we choose $\boldsymbol{P}$ as $\left[\begin{array}{lll}0 & 1 & 0\end{array}\right]^{T}$, which belongs to $\operatorname{Ker}\left(\boldsymbol{B}_{t \boldsymbol{k}}\right)$, the allocation input is thus constructed as $u_{t}=\boldsymbol{P} \boldsymbol{u}_{\boldsymbol{a}}$.

Define $\boldsymbol{Q} \triangleq\left[\begin{array}{ll}\boldsymbol{B}_{\boldsymbol{t} \boldsymbol{k}}^{T} & \boldsymbol{P}^{T}\end{array}\right]^{T}$ and $\boldsymbol{v} \triangleq \boldsymbol{Q} \boldsymbol{u}_{\boldsymbol{a}}=\left[\begin{array}{ll}\boldsymbol{v}_{\boldsymbol{t} \boldsymbol{k}}^{T} & u_{t}\end{array}\right]^{T}$. It is worth noting that $\operatorname{rank}(\boldsymbol{Q})=3$ and $\boldsymbol{u}_{\boldsymbol{a}}=\boldsymbol{Q}^{-1} \boldsymbol{v}$. Next, rewriting the actuator dynamics (2) in light of (8) yields

$$
\dot{x}_{a}=A_{v} x_{a}+B_{v a} u_{t}+R+B_{v d} d, y_{a}=C_{a} x_{a}
$$

where $A_{v} \triangleq A_{a}-B_{a} Q^{-1}\left[\begin{array}{lll}A_{x(2,6: 9)}^{T} & A_{x(5,6: 9)}^{T} & 0_{1 \times 4}^{T}\end{array}\right]^{T}, B_{v a} \triangleq$ $\boldsymbol{B}_{a} Q^{-1}\left[\begin{array}{lll}0 & 0 & 1\end{array}\right]^{T}, \boldsymbol{B}_{v d} \triangleq-\boldsymbol{B}_{a} \boldsymbol{Q}^{-1}\left[\boldsymbol{B}_{x d(2,:)}^{T} \boldsymbol{B}_{x d(5, ;)}^{T} \mathbf{0}_{\mathbf{1 \times 4}}^{T}\right]^{T}$ and $\boldsymbol{R} \triangleq \boldsymbol{B}_{\boldsymbol{a}} \boldsymbol{Q}^{-1}\left[u^{(2)}-\boldsymbol{A}_{\boldsymbol{x}(\mathbf{2 , 1 : 5})} \boldsymbol{D}_{\boldsymbol{Y}} h^{(3)}-\boldsymbol{A}_{\boldsymbol{x}(\mathbf{5 , 1 : 5})} \boldsymbol{D}_{\boldsymbol{Y}} 0\right]^{T}$.

The following assumption is adopted to facilitate the derivations, that is, in general, satisfied.

Assumption 2: $\left(A_{v}, B_{v a}\right)$ is controllable.

In what follows, the proposed dynamic allocation method is introduced, which is inspired by the generalized predictive control method [31]. However, unlike the generalized predictive control method, the desired states of actuators, rather than the states in (10), are optimized here. Note that in the output regulation framework for over-actuated systems [23]-[27], the desired states are also optimized based upon different cost functions.

\section{B. Generator and Estimator of Optimal Desired States}

The desired states of the considered actuators should satisfy the following system:

$$
\dot{x}_{a r}=A_{v} x_{a r}+B_{v a} u_{t r}+R_{r}+B_{v d} d, y_{a r}=C_{a} x_{a r} .
$$

If the tracking objective has been achieved, $\boldsymbol{R}_{\boldsymbol{r}}$ is also available as a priori knowledge, which is directly related to the reference commands, i.e., $\boldsymbol{R}_{\boldsymbol{r}}=\boldsymbol{B}_{\boldsymbol{a}} \boldsymbol{Q}^{-1}\left[u_{r}^{(2)}-\boldsymbol{A}_{\boldsymbol{x}(\mathbf{2 , 1 : 5 )}} \boldsymbol{D}_{\boldsymbol{Y} \boldsymbol{r}} h_{r}^{(3)}-\right.$ $\left.\boldsymbol{A}_{\boldsymbol{x}(\mathbf{5 , 1 : 5 )}} \boldsymbol{D}_{\mathbf{Y r}} 0\right]^{T}$.

Due to the linearization points (or trims) on the control inputs, the cost functions for each actuator can be defined as $J_{a}(t) \triangleq \int_{0}^{T}\left[\delta_{a r}(t+\tau)+\delta_{a}^{*}\right]^{2} d \tau, a \in\{e, t, f\}$, where $T>0$ is the predictive period. The total cost of actuators is defined as

$$
\begin{aligned}
J(t) & \triangleq \rho_{e} J_{e}(t)+\rho_{t} J_{t}(t)+\rho_{f} J_{f}(t) \\
& =\int_{0}^{T}\left[\boldsymbol{y}_{\boldsymbol{a r}}(t+\tau)+\boldsymbol{y}_{\boldsymbol{a}}^{*}\right]^{T} \boldsymbol{W}\left[\boldsymbol{y}_{\boldsymbol{a r}}(t+\tau)+\boldsymbol{y}_{\boldsymbol{a}}^{*}\right] d \tau
\end{aligned}
$$

where $\boldsymbol{y}_{\boldsymbol{a}}^{*} \triangleq\left[\begin{array}{lll}\delta_{e}^{*} & \delta_{t}^{*} & \delta_{f}^{*}\end{array}\right]^{T} ; \rho_{e}>0, \rho_{t}>0$ and $\rho_{f}>0$ are the weights on the elevator, motor throttle and flap, respectively, $\boldsymbol{W} \triangleq \operatorname{diag}\left(\rho_{e}, \rho_{t}, \rho_{d}\right)$. Inspired by [31], the following Taylor expansion with finite series is used to approximately predict $\boldsymbol{y}_{a r}(t+\tau)$ :

$\overline{\boldsymbol{y}}_{\boldsymbol{a r}}(t+\tau)=\boldsymbol{y}_{\boldsymbol{a r}}(t)+\tau \boldsymbol{y}_{\boldsymbol{a r}}^{(1)}(t)+\cdots+\frac{\tau^{n}}{n !} \boldsymbol{y}_{a r}^{(n)}(t), n \in \mathbb{N}_{+}, \tau \in[0, T]$

where $\overline{\boldsymbol{y}}_{\boldsymbol{a}}(t+\tau)$ is its prediction. Before optimization, notably, $C_{a} B_{v a}=0_{3 \times 1}$ and $C_{a} A_{v} B_{v a} \neq 0_{3 \times 1}$, which implies that the Taylor expansion order should be chosen as $n \geq 2, n \in$ $\mathbb{N}_{+}$. The case of $n=2$ is simple, i.e., $\boldsymbol{y}_{\boldsymbol{a r}}=\boldsymbol{C}_{\boldsymbol{a}} \boldsymbol{x}_{\boldsymbol{a}}$, $y_{a r}^{(1)}=C_{a} A_{v} x_{a r}+C_{a} R_{r}+C_{a} B_{v d} d$ and $y_{a r}^{(2)}=C_{a} A_{v}^{2} x_{a r}+$ $C_{a} A_{v} B_{v a} u_{t r}+C_{a} A_{v} R_{r}+C_{a} R_{r}^{(1)}+C_{a} A_{v} B_{v d} d$. Therefore, the detailed analysis of this case is ignored here.

Subsequently, a kind of compact optimization procedure is provided to simplify the general case of $n \geq 3, n \in \mathbb{N}_{+}$. First, extend the original system (11) by following integrators, and then regard $\boldsymbol{x}_{\boldsymbol{e r}} \triangleq\left[\begin{array}{llll}\boldsymbol{x}_{\boldsymbol{a r}}^{T} & u_{t r} & \cdots & u_{t r}^{(n-3)}\end{array}\right]^{T}$ as the new state of the extended system such that

$$
\dot{\boldsymbol{x}}_{\boldsymbol{e r}}=\boldsymbol{A}_{\boldsymbol{e}} \boldsymbol{x}_{\boldsymbol{e r}}+\boldsymbol{B}_{\boldsymbol{e v}} u_{t r}^{(n-2)}+\boldsymbol{B}_{\boldsymbol{e r}} \boldsymbol{R}_{\boldsymbol{r}}+\boldsymbol{B}_{\boldsymbol{e d}} \boldsymbol{d}, \boldsymbol{y}_{\boldsymbol{a r}}=\boldsymbol{C}_{\boldsymbol{e}} \boldsymbol{x}_{\boldsymbol{e r}}
$$

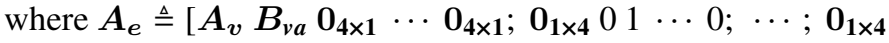
$\left.\begin{array}{lllllllllll}0 & 0 & \cdots & 1 ; & \mathbf{0}_{1 \times 4} & 0 & 0 & \cdots & 0\end{array}\right], \boldsymbol{B}_{\boldsymbol{e v}} \triangleq\left[\begin{array}{llllll}\mathbf{0}_{4 \times 1}^{T} & 0 & \cdots & 0 & 1\end{array}\right]^{T}, \boldsymbol{B}_{\boldsymbol{e r}} \triangleq$

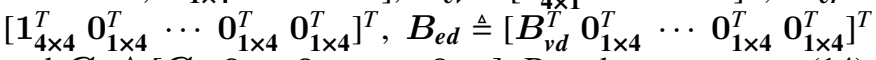
and $C_{e} \triangleq\left[\begin{array}{lllll}C_{a} & \mathbf{0}_{3 \times 1} & \mathbf{0}_{3 \times 1} & \cdots & \mathbf{0}_{3 \times 1}\end{array}\right]$. Based upon system (14), we have $\boldsymbol{y}_{a r}^{(1)}=\boldsymbol{C}_{\boldsymbol{e}} \boldsymbol{A}_{\boldsymbol{e}} \boldsymbol{x}_{\boldsymbol{e}}+\boldsymbol{C}_{\boldsymbol{e}} \boldsymbol{B}_{\boldsymbol{e}} \boldsymbol{R}_{\boldsymbol{r}}+\boldsymbol{C}_{\boldsymbol{e}} \boldsymbol{B}_{\boldsymbol{e}} \boldsymbol{d}, \cdots, \boldsymbol{y}_{a r}^{(n-1)}=$ $\boldsymbol{C}_{\boldsymbol{e}} \boldsymbol{A}_{\boldsymbol{e}}^{n-1} \boldsymbol{x}_{\boldsymbol{r}}+\sum_{i=0}^{n-2} \boldsymbol{C}_{\boldsymbol{e}} \boldsymbol{A}_{\boldsymbol{e}}^{i} \boldsymbol{B}_{\boldsymbol{e r}} \boldsymbol{R}_{\boldsymbol{r}}^{(n-2-i)}+\boldsymbol{C}_{\boldsymbol{e}} \boldsymbol{A}_{\boldsymbol{e}}^{n-2} \boldsymbol{B}_{\boldsymbol{e d}} \boldsymbol{d}$ and $\boldsymbol{y}_{a r}^{(n)}=C_{e} A_{e}^{n} x_{e r}+\sum_{i=0}^{n-1} C_{e} A_{e}^{i} B_{e r} R_{r}^{(n-1-i)}+C_{e} A_{e}^{n-1} B_{e d} d+$ $\boldsymbol{C}_{\boldsymbol{e}} \boldsymbol{A}_{\boldsymbol{e}}^{n-1} \boldsymbol{B}_{\boldsymbol{e v}} u_{t r}^{(n-2)}$. 
With the assistance of (13) and (14), the cost function (12) can be predicted as follows:

$$
\begin{aligned}
& \bar{J}(t)=\int_{0}^{T}\left[\overline{\boldsymbol{y}}_{a r}(t+\tau)+\boldsymbol{y}_{\boldsymbol{a}}^{*}\right]^{T} \boldsymbol{W}\left[\overline{\boldsymbol{y}}_{\boldsymbol{a r}}(t+\tau)+\boldsymbol{y}_{\boldsymbol{a}}^{*}\right] d \tau \\
& =u_{t r}^{(n-2) T} \mathcal{T}_{0} u_{t r}^{(n-2)}+2 \boldsymbol{x}_{\boldsymbol{e r}}^{T} \mathcal{T}_{1} u_{t r}^{(n-2)}+2 \boldsymbol{y}_{a}^{* T} \mathcal{T}_{2} u_{t r}^{(n-2)} \\
& +2\left[\begin{array}{llll}
\boldsymbol{R}_{\boldsymbol{r}}^{T} & \boldsymbol{R}_{\boldsymbol{r}}^{(1) T} & \cdots & \boldsymbol{R}_{\boldsymbol{r}}^{(n-1) T}
\end{array}\right] \mathcal{T}_{3} u_{t r}^{(n-2)}+2 \boldsymbol{d}^{T} \mathcal{T}_{4} u_{t r}^{(n-2)}+\star
\end{aligned}
$$

where $\mathcal{T}_{\mathbf{0}}$ to $\mathcal{T}_{4}$ are all collected in Appendix $\mathrm{B}$ and $\star$ is independent of $u_{t r}^{(n-2)}$. Letting $\partial \bar{J}(t) / \partial u_{t r}^{(n-2)}=0$ yields

$$
\begin{aligned}
u_{t r}^{(n-2)}=-\mathcal{T}_{0}^{-1}\left(\mathcal{T}_{1}^{T} \boldsymbol{x}_{e r}+\mathcal{T}_{2}^{T} \boldsymbol{y}_{\boldsymbol{a}}^{*}\right. & \\
& \left.+\mathcal{T}_{3}^{T}\left[\begin{array}{llll}
\boldsymbol{R}_{\boldsymbol{r}}^{T} \boldsymbol{R}_{\boldsymbol{r}}^{(1) T} & \cdots & \boldsymbol{R}_{r}^{(n-1) T}
\end{array}\right]^{T}+\mathcal{T}_{4}^{T} \boldsymbol{d}\right)
\end{aligned}
$$

which is an explicit solution of the optimal problem (12). Note that the existence of $\boldsymbol{d}$ in (14) and (16) makes the designed optimal desired states of actuators unimplementable, and hence, the construction of the estimates of these states should be considered first based upon observer (5), as follows:

$$
\begin{aligned}
& \dot{\hat{x}}_{e r}=A_{e} \hat{\boldsymbol{x}}_{e r}+\boldsymbol{B}_{e v} u_{t r}^{(\widehat{n-2})}+\boldsymbol{B}_{e r} \boldsymbol{R}_{r}+\boldsymbol{B}_{e d} \hat{\boldsymbol{d}} \\
& u_{t r}^{(n-2)}=-\mathcal{T}_{0}^{-1}\left(\mathcal{T}_{1}^{T} \hat{\boldsymbol{x}}_{e r}+\mathcal{T}_{2}^{T} \boldsymbol{y}_{a}^{*}\right. \\
& \left.+\mathcal{T}_{3}^{T}\left[\begin{array}{llll}
\boldsymbol{R}_{\boldsymbol{r}}^{T} & \boldsymbol{R}_{\boldsymbol{r}}^{(1) T} & \cdots & \boldsymbol{R}_{\boldsymbol{r}}^{(n-1) T}
\end{array}\right]^{T}+\mathcal{T}_{4}^{T} \hat{d}\right)
\end{aligned}
$$

where $\hat{\boldsymbol{x}}_{\boldsymbol{e r}} \triangleq\left[\begin{array}{llll}\hat{\boldsymbol{x}}_{a r}^{T} & \hat{u}_{t r} & \cdots & \widehat{u_{t r}^{(n-3)}}\end{array}\right]^{T}$.

Remark 2: The main computational burden of the dynamic control allocation method is to compute matrices $\mathcal{T}_{0}$ to $\mathcal{T}_{4}$ in (15). In principle, as long as the Taylor expansion order $n$ is fixed, all computation can be done off-line, which means that matrices $\mathcal{T}_{0}$ to $\mathcal{T}_{4}$ are explicit with respect to the predictive period $T$ and weighting matrix $\boldsymbol{W}$, making the real-time tuning available.

Remark 3 (Modular Design): Optimizing the desired states of actuator $x_{a r}$, rather than directly optimizing the state $\boldsymbol{x}_{\boldsymbol{a}}$, is adopted here to pursue modular design. Replacing $\boldsymbol{R}_{\boldsymbol{r}}$ in (16) with $\boldsymbol{R}$ in (10), one will find that once trying to directly optimize the actuator states, higher order observer should be redesigned to estimate $\boldsymbol{R}, \boldsymbol{R}^{(1)}, \cdots, \boldsymbol{R}^{(n-1)}$. This implies that the whole control structure will be changed as long as the Taylor expansion order $n$ in (13) is changed.

\section{Dynamic Control Allocator Design}

In this step, the control input $u_{t}$ is designed to force the real actuator state $\boldsymbol{x}_{\boldsymbol{a}}$ to the optimal one $\boldsymbol{x}_{\boldsymbol{a r}}$. Thus, we can design the following allocator:

$$
u_{t}=\boldsymbol{K}_{t}\left(\hat{\boldsymbol{x}}_{\boldsymbol{a r}}-\hat{\boldsymbol{x}}_{\boldsymbol{a}}\right)+\hat{u}_{t r}
$$

where $\boldsymbol{K}_{\boldsymbol{t}}$ is the allocator gain. Based upon the preceding tracking controller (9) and allocator (18), the physical controller $\boldsymbol{u}_{\boldsymbol{a}}$ is obtained as

$$
u_{a}=Q^{-1} v
$$

\section{Performance Analysis}

Theorem 1: Under Assumptions 1 and 2, consider the closed-loop system (1)-(2)-(19). If all the control parameters are well tuned, i.e., for observer (5), $\boldsymbol{A}_{\boldsymbol{a d}}-\boldsymbol{L} \boldsymbol{C}_{\boldsymbol{a d}}$ is Hurwitz; for controller (9), [0 1; 0 0 $]-\left[\begin{array}{ll}0 & 1\end{array}\right]^{T} \boldsymbol{K}_{\boldsymbol{u}}$ and

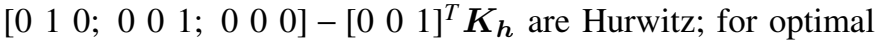
desired state generator (17), $\boldsymbol{A}_{\boldsymbol{e}}-\boldsymbol{B}_{\boldsymbol{e v}} \mathcal{T}_{0}^{-1} \mathcal{T}_{1}^{T}$ is Hurwitz; for allocator (18), $\boldsymbol{A}_{\boldsymbol{v}}-\boldsymbol{B}_{v a} \boldsymbol{K}_{\boldsymbol{t}}$ is Hurwitz, then the following three statements hold:

1) Uniform Boundedness: All the signals in the closed-loop system are uniformly bounded.

2) Asymptotic Tracking: The output of the UAV $\boldsymbol{y}_{p}$ asymptotically tracks any given sufficiently smooth and bounded reference command $\boldsymbol{y}_{\boldsymbol{p}}$, i.e., $\lim _{t \rightarrow+\infty} \boldsymbol{y}_{\boldsymbol{p}}=\boldsymbol{y}_{\boldsymbol{p} r}$.

3) Control Allocation: The state of the actuator $\boldsymbol{x}_{\boldsymbol{a}}$ asymptotically tracks the solution of the optimal problem $x_{a r}$, which is generated by (14) and (16), i.e., $\lim _{t \rightarrow+\infty} x_{a}=x_{a r}$. Proof: Define the estimation errors as $\boldsymbol{e}_{\boldsymbol{a d}} \triangleq \boldsymbol{x}_{\boldsymbol{a d}}-\hat{\boldsymbol{x}}_{\boldsymbol{a d}}$ and $\boldsymbol{e}_{\boldsymbol{r}} \triangleq \boldsymbol{x}_{\boldsymbol{r} r}-\hat{\boldsymbol{x}}_{\boldsymbol{r} r}$. Define the tracking errors as $\boldsymbol{E}_{\boldsymbol{a}} \triangleq \boldsymbol{x}_{\boldsymbol{a r}}-\boldsymbol{x}_{\boldsymbol{a}}$, $\boldsymbol{E}_{u} \triangleq\left[u_{r}-u u_{r}^{(1)}-u^{(1)}\right]^{T}$ and $\boldsymbol{E}_{\boldsymbol{h}} \triangleq\left[h_{r}-h h_{r}^{(1)}-h^{(1)} h_{r}^{(2)}-h^{(2)}\right]^{T}$. Omitting tedious derivation gives the following error system:

$$
\begin{aligned}
{\left[\begin{array}{c}
\dot{E}_{a} \\
\dot{E}_{u} \\
\dot{E}_{h} \\
\dot{e}_{e r} \\
\dot{e}_{a d}
\end{array}\right]=} & {\left[\begin{array}{ccc}
A_{v}-B_{v a} K_{t} & \Lambda_{1} & \Lambda_{2} \\
0_{2 \times 4} & A_{K_{u}} & 0_{2 \times 3} \\
0_{3 \times 4} & 0_{3 \times 2} & A_{K_{h}} \\
0_{(n+2) \times 4} & 0_{(n+2) \times 2} & 0_{(n+2) \times 3} \\
0_{8 \times 4} & 0_{8 \times 2} & 0_{8 \times 3} \\
\Lambda_{3} & \Lambda_{4} \\
0_{2 \times(n+2)} & \Lambda_{5} \\
0_{3 \times(n+2)} & \Lambda_{6} \\
A_{e}-B_{e v} \mathcal{T}_{0}^{-1} \mathcal{T}_{1}^{T} & \Lambda_{7} \\
0_{8 \times(n+2)} & A_{a d}-L C_{a d}
\end{array}\right]\left[\begin{array}{c}
E_{a} \\
E_{u} \\
E_{h} \\
e_{e r} \\
e_{a d}
\end{array}\right] }
\end{aligned}
$$

where $\boldsymbol{A}_{\boldsymbol{K}_{u}} \triangleq\left[\begin{array}{llll}0 & 1 ; & 0 & 0\end{array}\right]-\left[\begin{array}{ll}0 & 1\end{array}\right]^{T} \boldsymbol{K}_{\boldsymbol{u}}, \quad \boldsymbol{A}_{\boldsymbol{K}_{h}} \triangleq$ $\left[\begin{array}{llllllll}0 & 1 & 0 & 0 & 0 & 1\end{array} ; 0000\right]-\left[\begin{array}{llll}0 & 0 & 1\end{array}\right]^{T} \boldsymbol{K}_{\boldsymbol{h}}$ and $\boldsymbol{\Lambda}_{\boldsymbol{i}}(i=1,2, \cdots, 7)$ are nonzero matrixes. If all the control parameters are well tuned, system (20) is globally asymptotically stable. Besides, since that $u_{r}, u_{r}^{(1)}, u_{r}^{(2)}, h_{r}, h_{r}^{(1)}, h_{r}^{(2)}, h_{r}^{(3)}$ and $\boldsymbol{d}$ are all bounded, all the signals in the closed-loop system are uniformly bounded.

This completes the proof of Theorem 1.

Remark 4 (Hierarchy Parameter Tuning): From dynamics of the output tracking errors, $\boldsymbol{E}_{\boldsymbol{u}}$ and $\boldsymbol{E}_{\boldsymbol{h}}$, one can conclude that the tracking performance in high level is segregated from the allocation performance in low level. And hence, the tuning process can also be divided as two steps. First, tune parameters of the proposed actuator/disturbance observer (5) and tracking controller (9) to satisfy specific requirements on the output tracking. Second, tune the weighting matrix $W$ to penalize the specific actuator.

\section{Simulation Study}

In this section, X-Plane flight simulation software is employed to verify the proposed dynamic control allocation method. X-Plane is popular in aeronautical engineering due to its high fidelity on both aircraft dynamics and atmospheric environment [38]. In particular, the simulations are conducted based upon a geometrical UAV model built in X-Plane 
using blade element theory to determine the aerodynamic performance. The considered UAV in X-Plane is illustrated in Fig. 4. To generate extra disturbances and uncertainties in the simulation, we have added $2 \mathrm{~m} / \mathrm{s}$ wind gusts as well as small changes on the centre of gravity and weight of the aircraft. The constraints on the outputs of actuators are chosen as $\delta_{e}+\delta_{e}^{*} \in\left[-50^{\circ}, 50^{\circ}\right], \delta_{f}+\delta_{f}^{*} \in\left[-50^{\circ}, 50^{\circ}\right]$ and $\delta_{t}+\delta_{t}^{*} \in[0,100 \%]$.

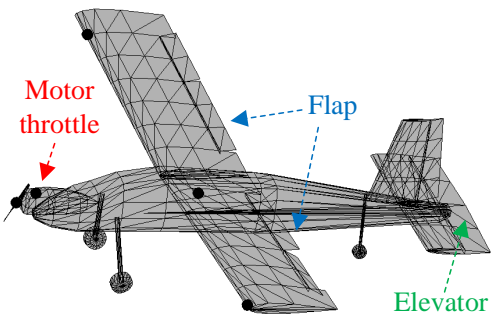

Fig. 4. The 3D model of the used UAV in X-Plane.

To demonstrate the effectiveness of the proposed method, both the conventional static control allocation (CSCA) method [8] and the conventional dynamic control allocation (CDCA) method [21] are adopted as benchmarks. Physical constraints are considered in both CSCA and CDCA methods whilst actuator dynamics are only used in the design of CDCA and the proposed methods. The high-level tracking controllers are also designed following the same approach. Meanwhile, for fair comparisons, the controller gains of the three methods are tuned to render similar offset errors when the disturbance estimates are not included in the controllers, as shown in the gray patches of the subsequent simulation results. The observer gains are then fixed by making the poles of estimation error system five times of those of tracking error systems. It should be noted that although the CDCA method is able to deal with actuator dynamics, this method replies on the direct measurement of the actuator states, hence is not applicable in practice.

In what follows, two case studies are carried out to show the improvement of the proposed method on robustness and disturbance rejection.

\section{A. Robustness Improvement}

Following the test approaches of control allocation methods considering actuator dynamics [20], [21], [39], we also consider a sine signal with time-increasing frequency as the reference of the velocity but a constant reference of the height, which are both depicted in the red dash lines of Fig. 5 (a). The output tracking results of the UAV and the outputs of the actuators under the different allocation methods, and the estimates of the total disturbances are illustrated in Fig. 5 (a), Fig. 5 (b) and Fig. 5 (c), respectively. It can be observed from the velocity tracking result in Fig. 5 (a), the disturbance observer will improve the robustness of the proposed method against the unmodelled dynamics of both aircraft dynamics and atmospheric environment. Moreover, the height tracking precision of the proposed method is much higher than those of the CSCA and CDCA methods when the disturbance estimates are included. The main reason is that without considering the compensation for the lag effect of the actuators, the tracking error will largely depend on the frequency of its reference, especially when the frequency beyond the bandwidth. After nearly 70s, the UAV under the CSCA method tends to be unstable. The essential reason is that the CSCA method is not able to actively stabilize the zero dynamics [30], and hence, its control parameters are very sensitive. Although the UAV under the CSCA method works well at the beginning, it inevitably becomes unstable when the unmodelled dynamics becomes more significant, e.g., in the case where the reference changes more dramatically.

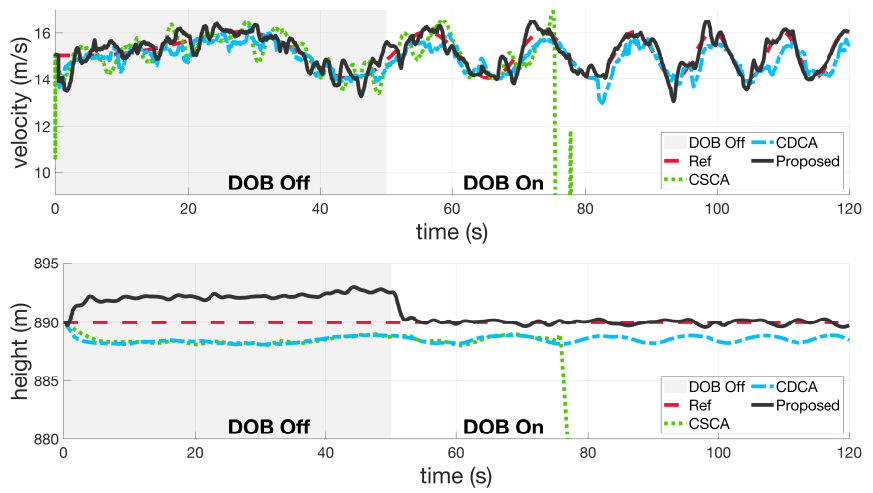

(a)
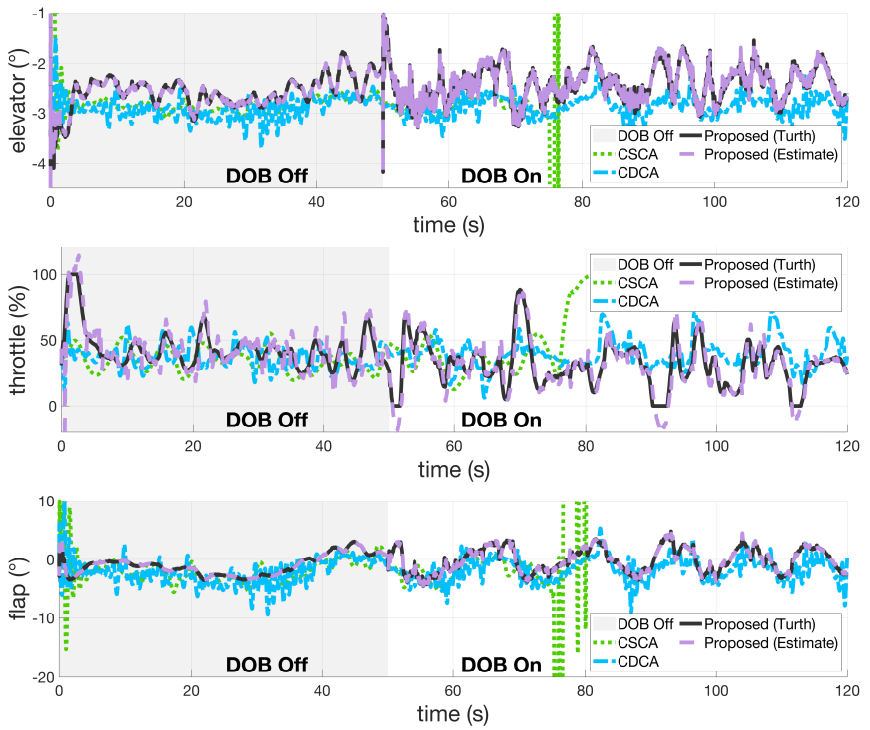

(b)

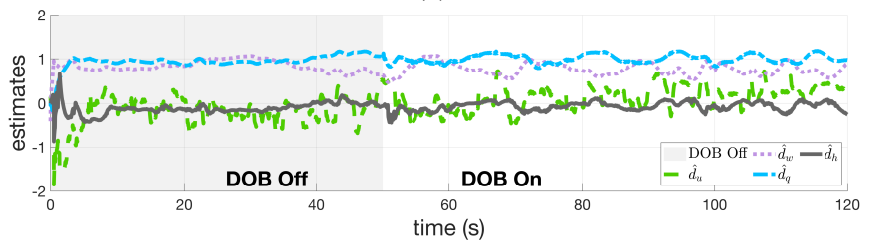

(c)

Fig. 5. Tracking performance under the sine reference with time-increasing frequency. (a): Outputs of UAV. (b): Outputs and estimates of actuators. (c): Estimates of disturbances. 


\section{B. Disturbance Rejection}

In the second case study ${ }^{1}$, in the presence of additional wind gusts, the UAV is controlled to track a descending profile with airspeed reduced from $15 \mathrm{~m} / \mathrm{s}$ to $12 \mathrm{~m} / \mathrm{s}$ and height from $890 \mathrm{~m}$ to $840 \mathrm{~m}$, which are depicted in the red dash lines of Fig. 6 (a). Due to the weakness of the CSCA method in the first case study, the comparison with only the CDCA method is implemented here. The output tracking results of the UAV, the outputs and estimates of the actuators, and the estimates of the total disturbances are illustrated in Fig. 6 (a), Fig. 6 (b) and Fig. 6 (c), respectively. It can be observed from these tracking results, the proposed method will largely improve the tracking precisions compared with the CDCA method, which is critical for UAV safety under wind conditions. Since that total disturbances are still unmeasurable even in X-Plane simulation, the estimation performance of disturbances can be indirectly verified by the improvement of tracking precisions in Fig. 6 (a) and the estimation precisions of actuators in Fig. 6 (b). From Fig. 6 (c), the estimate of $d_{u}$ changes relatively fast but is very small around zero whilst the estimates of $d_{w}, d_{q}$ and $d_{h}$ are all slowly time-varying changing.

\section{Conclusion}

In this work, a new actuator-dynamics-based dynamic control allocation scheme is developed for flight control of a small fixed-wing UAV with DLC to compensate unknown external disturbances. Compared to the conventional UAVs, the considered small UAV presents faster dynamics, hence the actuator dynamics cannot be ignored in high-precision applications. By embedding the internal models of actuators into both the tracking controller design in the high level and dynamic allocator design in the low level, remarkable superiorities in tracking precision of UAVs and energy efficiency can be achieved by the proposed method, which have been demonstrated in the verification example using high fidelity simulations. It is envisaged that the proposed control scheme can be used to improve the flight performance and extend the flight envelop of small fixed-wing UAVs with dedicated flaps, allowing them to be used in wider applications and unfavourable weather conditions. Future work will be focusing on the extension of the current framework to deal with actuator constraints.

\section{APPENDIX}

\section{A. Parameters}

The UAV model parameters are obtained through system identification around the operation point of level flight at forward velocity $15 \mathrm{~m} / \mathrm{s}$ and height $890 \mathrm{~m}$, whereas the actuator dynamics are established based upon the ground test data of the servos used on the UAV at the sampling rate of $100 \mathrm{~Hz}$. The identification process is detailed in [40, Chap. 4]. The sampling rate of the control program is $50 \mathrm{~Hz}$. For the sake of completeness, the UAV parameters are listed in Table I.

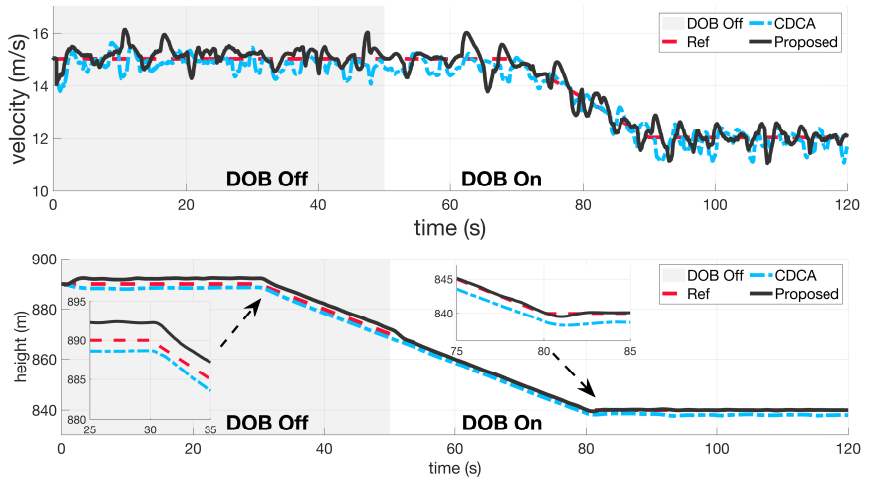

(a)
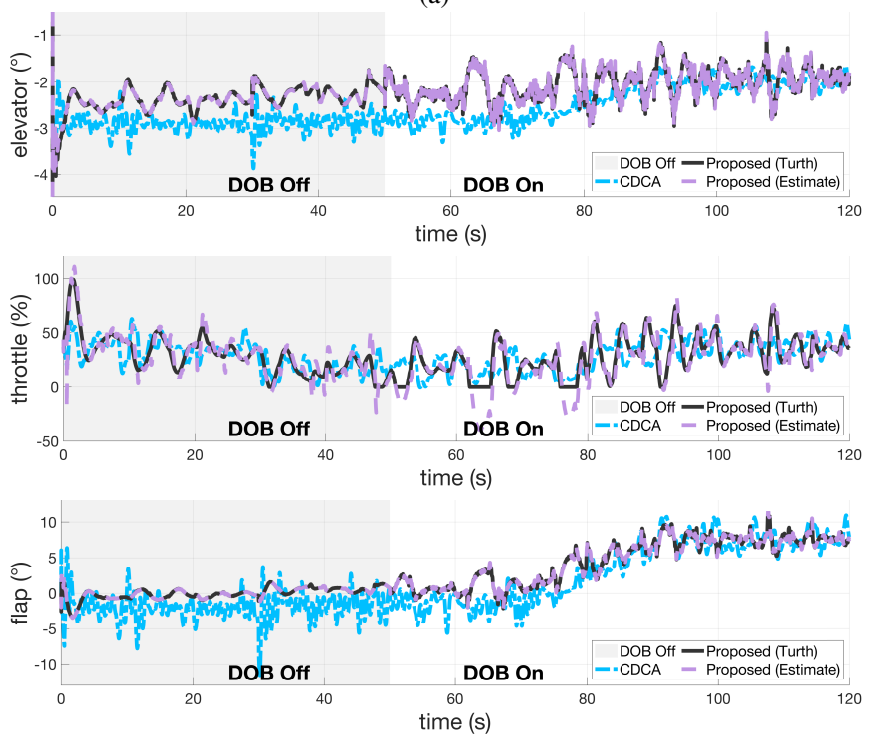

(b)

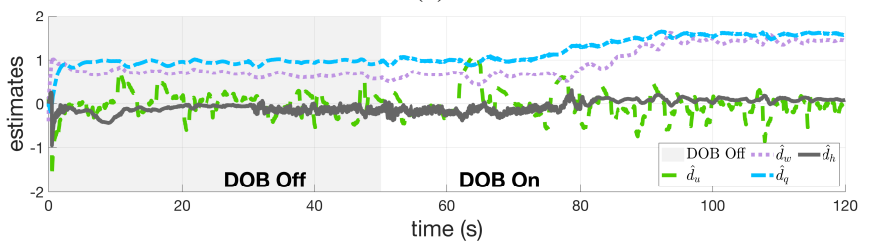

(c)

Fig. 6. Tracking performance in the presence of wind gusts. (a): Outputs of UAV. (b): Outputs and estimates of actuators. (c): Estimates of disturbances.

TABLE I

Parameters of the UAV and the Actuators

\begin{tabular}{ll}
\hline Parameter & Value \\
\hline$X_{u}, X_{w}, X_{q}, X_{\delta_{e}}, X_{\delta_{t}}, X_{\delta_{d}}$ & $-0.116,0.922,-0.352,0,2.72,-0.029$ \\
$Z_{u}, Z_{w}, Z_{q}, Z_{\delta_{e}}, Z_{\delta_{d}}$ & $-1.05,-6.74,14.99,-0.095,-0.34$ \\
$M_{u}, M_{w}, M_{q}, M_{\delta_{e}}, M_{\delta_{d}}$ & $0.144,-3.87,-3.8,-1.99,0.187$ \\
$u^{*}, w^{*}, q^{*}, \theta^{*}, h^{*} ; \delta_{e}^{*}, \delta_{t}^{*}, \delta_{f}^{*}$ & $15.04,0.57,0,0.038,890 ;-2.7,0.37,0$ \\
$\tau_{e}, \tau_{d}, \zeta_{t}, \omega_{n t}$ & $0.16,0.16,1,1.6$ \\
\hline
\end{tabular}

\section{B. Expressions}

The expressions of $\mathcal{T}_{\mathbf{0}}$ to $\mathcal{T}_{\mathbf{4}}$ are presented as follows: 


$$
\begin{aligned}
& \mathcal{T}_{0} \triangleq \int_{0}^{T}\left(\frac{\tau^{n}}{n !} C_{e} A_{e}^{n-1} B_{e v}\right)^{T} \boldsymbol{W}\left(\frac{\tau^{n}}{n !} C_{e} A_{e}^{n-1} B_{e v}\right) d \tau \\
& \mathcal{T}_{\mathbf{1}} \triangleq \int_{0}^{T}\left(C_{e}+\cdots+\frac{\tau^{n}}{n !} C_{e} A_{e}^{n}\right)^{T} \boldsymbol{W}\left(\frac{\tau^{n}}{n !} C_{e} A_{e}^{n-1} B_{e v}\right) d \tau \\
& \mathcal{T}_{2} \triangleq \int_{0}^{T} \boldsymbol{W}\left(\frac{\tau^{n}}{n !} C_{e} A_{e}^{n-1} B_{e v}\right) d \tau \\
& \mathcal{T}_{\mathbf{3}} \triangleq\left[\begin{array}{llll}
\mathcal{T}_{\mathbf{3 1}}^{T} & \mathcal{T}_{\mathbf{3 2}}^{T} & \cdots & \mathcal{T}_{\mathbf{3 n}}^{T}
\end{array}\right]^{T} \\
& \mathcal{T}_{3 i} \triangleq \int_{0}^{T}\left(\tau C_{e} B_{e r}+\cdots+\frac{\tau^{n}}{n !} C_{e} A_{e}^{n-i} B_{e r}\right)^{T} W \\
& \times\left(\frac{\tau^{n}}{n !} \boldsymbol{C}_{e} \boldsymbol{A}_{e}^{n-1} \boldsymbol{B}_{\boldsymbol{e} v}\right) d \tau, i=1,2, \cdots, n \\
& \mathcal{T}_{4} \triangleq \int_{0}^{T}\left(\tau C_{e} B_{e d}+\cdots+\frac{\tau^{n}}{n !} C_{e} A_{e}^{n-1} B_{e d}\right)^{T} W \\
& \times\left(\frac{\tau^{n}}{n !} C_{e} A_{e}^{n-1} B_{e v}\right) d \tau .
\end{aligned}
$$

\section{REFERENCES}

[1] P. Poksawat, L. Wang, and A. Mohamed, "Gain scheduled attitude control of fixed-wing UAV with automatic controller tuning," IEEE Trans. Control Syst. Technol., vol. 26, no. 4, pp. 1192-1203, Jul. 2018.

[2] C. Liu and W.-H. Chen, "Disturbance rejection flight control for small fixed-wing unmanned aerial vehicles," J. Guid. Control Dyn., pp. 28102819, Dec. 2016.

[3] Y. Luo, H. Chao, L. Di, and Y. Chen, "Lateral directional fractional order $(\mathrm{PI})^{\alpha}$ control of a small fixed-wing unmanned aerial vehicles: Controller designs and flight tests," IET Contr. Theory Appl., vol. 5, no. 18, pp. 2156-2167, May. 2011.

[4] C. Liu, O. McAree, and W.-H. Chen, "Path-following control for small fixed-wing unmanned aerial vehicles under wind disturbances," Int. J. Robust Nonlinear Control, vol. 23, no. 15, pp. 1682-1698, Dec. 2013.

[5] O. Lykken and N. M. Shan, "Direct lift control for improved automatic landing and performance of transport aircraft," J. Aircr., vol. 9, no. 5, pp. 325-332, May. 1972.

[6] M. Bodson, "Evaluation of optimization methods for control allocation," J. Guid. Control Dyn., vol. 25, no. 4, pp. 703-711, Jul./Aug. 2002.

[7] T. A. Johansen and T. I. Fossen, "Control allocation-A survey," Automatica, vol. 49, no. 5, pp. 1087-1103, Mar. 2013.

[8] O. Härkegård, "Efficient active set algorithms for solving constrained least squares problems in aircraft control allocation," in 41st IEEE Conf. Decision and Control (CDC), Las Vegas, Newla USA, Dec. 2002.

[9] — - "Backstepping and control allocation with applications to flight control," Ph.D. dissertation, Linköpings Univ., 2003.

[10] G. De Tommasi, S. Galeani, A. Pironti, G. Varano, and L. Zaccarian, "Nonlinear dynamic allocator for optimal input/output performance trade-off: Application to the jet tokamak shape controller," Automatica, vol. 47, no. 5, pp. 981-987, Feb. 2011.

[11] J.-F. Trégouët, D. Arzelier, D. Peaucelle, C. Pittet, and L. Zaccarian, "Reaction wheels desaturation using magnetorquers and static input allocation," IEEE Trans. Control Syst. Technol., vol. 23, no. 2, pp. 525539, Mar. 2015

[12] A. Mark, Y. Xu, and B. T. Dickinson, "Consensus-based decentralized aerodynamic moment allocation among synthetic jets and control surfaces," IEEE Trans. Control Syst. Technol., doi:10.1109/TCST.2018.2859908

[13] O. Härkegård and S. T. Glad, "Resolving actuator redundancy—Optimal control vs. control allocation," Automatica, vol. 41, no. 1, pp. 137-144, Oct. 2005.

[14] J. A. M. Petersen and M. Bodson, "Constrained quadratic programming techniques for control allocation," IEEE Trans. Control Syst. Technol., vol. 14, no. 1, pp. 91-98, Jan. 2006.

[16] M. Kirchengast, M. Steinberger, and M. Horn, "Input matrix factorizations for constrained control allocation," IEEE Trans. Autom. Control, vol. 63, no. 4, pp. 1163-1170, Apr. 2018.
[15] L. Zaccarian, "Dynamic allocation for input redundant control systems," Automatica, vol. 45, no. 6, pp. 1431-1438, Mar. 2009.

[17] O. Härkegård, "Dynamic control allocation using constrained quadratic programming," J. Guid. Control Dyn., vol. 27, no. 6, pp. 1028-1034, Nov./Dec. 2004

[18] Q. Hu, B. Li, and Y. Zhang, "Nonlinear proportional-derivative control incorporating closed-loop control allocation for spacecraft," J. Guid. Control Dyn., vol. 37, no. 3, pp. 799-812, May./Jun. 2014.

[19] M. E. Raoufat, K. Tomsovic, and S. M. Djouadi, "Dynamic control allocation for damping of inter-area oscillations," IEEE Trans. Power Syst., vol. 32, no. 6, pp. 4894-4903, Nov. 2017.

[20] M. W. Oppenheimer and D. B. Doman, "Methods for compensating for control allocator and actuator interactions," J. Guid. Control Dyn., vol. 27, no. 5, pp. 922-927, Apr. 2004.

[21] M. Hanger, T. A. Johansen, G. K. Mykland, and A. Skullestad, "Dynamic model predictive control allocation using CVXGEN," in 9th IEEE Int. Conf. Control Autom. (ICCA), Santiago, Chile, Dec. 2011.

[22] J. Tjønnås and T. A. Johansen, "Adaptive control allocation," Automatica, vol. 44, no. 11, pp. 2754-2765, Oct. 2008.

[23] A. Serrani, "Output regulation for over-actuated linear systems via inverse model allocation," in 51st IEEE Conf. Decision and Control $(C D C)$, Maui, Hawaii, USA, Dec. 2012.

[24] S. Galeani, A. Serrani, G. Varano, and L. Zaccarian, "On input allocation-based regulation for linear over-actuated systems," Automat$i c a$, vol. 52, pp. 346-354, Jan. 2015.

[25] M. Cocetti, A. Serrani, and L. Zaccarian, "Linear output regulation with dynamic optimization for uncertain linear over-actuated systems," Automatica, vol. 97, pp. 214-225, Jun. 2018.

[26] J. Zhou, M. Canova, and A. Serrani, "Predictive inverse model allocation for constrained over-actuated linear systems," Automatica, vol. 67, pp. 267-276, Feb. 2016.

[27] _ "Non-intrusive reference governors for over-actuated linear systems," IEEE Trans. Autom. Control, vol. 62, no. 9, pp. 4734-4740, Sep. 2017.

[28] W.-H. Chen, J. Yang, L. Guo, and S. Li, "Disturbance-observer-based control and related methods-An overview," IEEE Trans. Ind. Electron., vol. 63, no. 2, pp. 1083-1095, Feb. 2016.

[29] M. Benosman, F. Liao, K.-Y. Lum, and J. L. Wang, "Nonlinear control allocation for non-minimum phase systems," IEEE Trans. Control Syst. Technol., vol. 17, no. 2, pp. 394-404, Mar. 2009.

[30] J. M. Buffington, D. F. Enns, and A. R. Teel, "Control allocation and zero dynamics," J. Guid. Control Dyn., vol. 21, no. 3, pp. 458-464, May./Jun. 1998.

[31] W.-H. Chen, D. J. Ballance, and P. J. Gawthrop, "Optimal control of nonlinear systems: A predictive control approach," Automatica, vol. 39, no. 4, pp. 633-641, Apr. 2003.

[32] S. S. Mulgund and R. F. Stengel, "Optimal nonlinear estimation for aircraft flight control in wind shear," Automatica, vol. 32, no. 1, pp. 3-13, May. 1996.

[33] R. W. Beard and T. W. McLain, Small unmanned aircraft: Theory and practice. Princeton, New Jersey, USA: Princeton University Press, 2012.

[34] B. K. Ghosh and J. Rosenthal, "A generalized Popov-Belevitch-Hautus test of observability," IEEE Trans. Autom. Control, vol. 40, no. 1, pp. 176-180, Jan. 1995.

[35] W.-H. Chen, "Disturbance observer based control for nonlinear systems," IEEE/ASME Trans. Mechatron., vol. 9, no. 4, pp. 706-710, Dec. 2004.

[36] A. Isidori, Nonlinear Control Systems (Communications and Control Engineering). London, U.K.: Springer-Verlag, 1995.

[37] S. H. Lane and R. F. Stengel, "Flight control design using non-linear inverse dynamics," Automatica, vol. 24, no. 4, pp. 471-483, Jan. 1988.

[38] J. Craighead, R. Murphy, J. Burke, and B. Goldiez, "A survey of commercial \& open source unmanned vehicle simulators," in 24th IEEE Int. Conf. Robot. Autom (ICRA), Roma, Italy, Apr. 2007.

[39] S. Sen, G. Ray, and T. K. Ghoshal, "Dynamic control allocation for tracking time-varying control demand," J. Guid. Control Dyn., vol. 31, no. 4, pp. 1150-1157, Jul./Aug. 2008.

[40] J. Smith, "Design and application of advanced disturbance rejection control for small fixed-wing UAVs," Ph.D. dissertation, Loughborough University, 2018. 